\title{
Smear-Negative Pulmonary Tuberculosis
}

\author{
Tayfun Çalışkan ${ }^{1}$, Hatice Kaya²
}

${ }^{1}$ Clinic of Chest Diseases, Gülhane Military Medical Academy Haydarpaşa Training Hospital, İstanbul

${ }^{2}$ Clinic of Chest Diseases, Gülhane Military Medical Academy, Ankara

\begin{abstract}
Tuberculosis (TB) is the second leading cause of death from an infectious disease worldwide, after the human immunodeficiency virus. There are almost 2.5 million new smear-positive pulmonary TB cases and 1.9 million new smear-negative pulmonary TB cases in the world in 2012 according to the World Health Organization (WHO) Global Tuberculosis Report 2013. Smear negativity in pulmonary TB is a common clinical problem. Clinicians have difficulty in diagnosing smear-negative pulmonary TB without bacteriological confirmation. It is very important to decide whether or not to treat a patient with smear negative pulmonary TB when the culture results are pending or negative. New diagnostic methods are required for the diagnosis of smear-negative pulmonary TB. In addition to the development of new microbiological and serological diagnostic tests, clinical prediction scoring systems and algorithms including clinical and radiological findings of smear-negative pulmonary TB patients in our country, should be established to facilitate the diagnosis of smear-negative pulmonary TB.
\end{abstract}

Keywords: Pulmonary, smear negative tuberculosis, tuberculosis

Received Date: 02.05.2014 Accepted Date: 04.08.2014

Address for correspondence Tayfun Çalışkan

E-mail: drtcaliskan@yahoo.com

This work is licensed under a Creative Commons Attribution-NonCommercia 4.0 International License.

DOI: 10.5152/ejp.2014.89106

-Available online at www.eurasianjpulmonol.com

\section{INTRODUCTION}

Pulmonary tuberculosis is still one of the most common infectious diseases in the world. According to the Tuberculosis Control Report published by World Health Organization (WHO) in 2013, approximately 2.5 million new smear-positive pulmonary tuberculosis and 1.9 million new smear-negative pulmonary tuberculosis (SNPT) patients were reported (1). It is seen that among new cases, the ratio of smear-positive pulmonary tuberculosis is $57 \%$ and most patients are SNPT.

When the data from Turkey were examined, 4595 new cases of smear-positive pulmonary tuberculosis and 3829 new cases of SNPT or smear-unknown pulmonary tuberculosis were detected in 2012 (2). Among new cases of pulmonary tuberculosis, the rate of smear-positive pulmonary tuberculosis is 54\%. In Turkey, according to the 2012 Tuberculosis Control Report, $60.1 \%$ of the total pulmonary tuberculosis patients have smear-positive pulmonary tuberculosis, $28.5 \%$ have SNPT and $11.4 \%$ have smear-unknown pulmonary tuberculosis. The rate of smear-unknown pulmonary tuberculosis cases, which were $20.8 \%$ in 2005 , has decreased with time (2). However, a relative increase has been observed in the following years in SNPT cases, whose rate was $22.4 \%$ in 2005.

\section{Definition}

There are serious unsolved problems regarding the definition and, accordingly, the diagnosis of SNPT. There are different recommendations in various guidelines. According to the WHO definition, SNPT is defined as follows:

a. Pulmonary tuberculosis cases with negative sputum smears but growth positive in cultures;

b. The patients who meet all of the four criteria stated below:

1. At least two negative sputum smears,

2. Radiological findings consistent with radiologically active pulmonary tuberculosis,

3. No response to a course of broad-spectrum antibiotics (except patients with clinically validated HIV infection or with strong clinical findings suggesting a HIV infection),

4. A decision made by a clinician for the treatment with antituberculosis medication (1). 
According to the Tuberculosis Diagnosis and Treatment Guideline published in Turkey 2011, SNPT is present in patients who have the following:

a. Negative sputum smears but growth positive in cultures,

b. Clinical and radiological findings consistent with tuberculosis, three negative sputum smears, and a decision of tuberculosis treatment in a hospital with opportunities for a differential diagnosis after no response has been obtained despite the use of broad-spectrum antibiotics (without quinolone) for at least a week (3).

There are differences as well as similarities in the two definitions. First, although WHO includes at least two smear-negativities in the definition, the Turkey guideline states that at least three smear-negativities are required. SNPT, which is defined as patients with smear-negative and with findings radiologically consistent with pulmonary tuberculosis in WHO, guideline is defined as patients with findings clinically and radiologically consistent with pulmonary tuberculosis in the guidelines of Turkey. For HIV-negative patients with a pre-diagnosis of SNPT, antituberculosis treatment should be initiated following the elimination of other pulmonary infections according to the response on the clinical and control chest X-ray after taking at least two sputum samples on different days and the use of quinolone-free antibiotics for a week on average (5-30 days).
In patients with a pre-diagnosis of SNPT, the selection and period of the antibiotic treatment is controversial. In antibiotic selection, the use of fluoroquinolones should be avoided because of the antituberculosis effectiveness. Samples for the causes of non-tuberculosis pulmonary infection should be taken from patients and further investigations should be performed. Trial treatments with antituberculosis medication are not recommended for patients with a pre-diagnosis of SNPT because the factors of the non-tuberculosis infection can respond to this treatment. The algorithm suggested by WHO for SNPT diagnosis in HIV-negative patients is shown in Figure 1 (4).

Smear-negative pulmonary tuberculosis occurs less frequently than smear-positive pulmonary tuberculosis, but it is an infectious communicable form of tuberculosis. In a cohort study conducted in Holland, it was revealed that smear-negative culture-positive pulmonary tuberculosis was responsible for $13 \%$ of the contagion of tuberculosis (5). In another study conducted in San Francisco, it was detected that at least $17 \%$ of the contagion of tuberculosis was due to SNPT (6). SNPT is an infectious and a communicable contagious disease. SNPT and extrapulmonary tuberculosis are responsible for $13-41 \%$ of the tuberculosis contagion (7).

Smear-negative pulmonary tuberculosis is differentiated from smear-positive pulmonary tuberculosis in some aspects. There are 1.22 SNPT and extrapulmonary tuberculosis cases per one smear-positive pulmonary tuberculosis patient in the society (8). In SNPT, minimal disease is more frequent because of small amount of bacillary load,

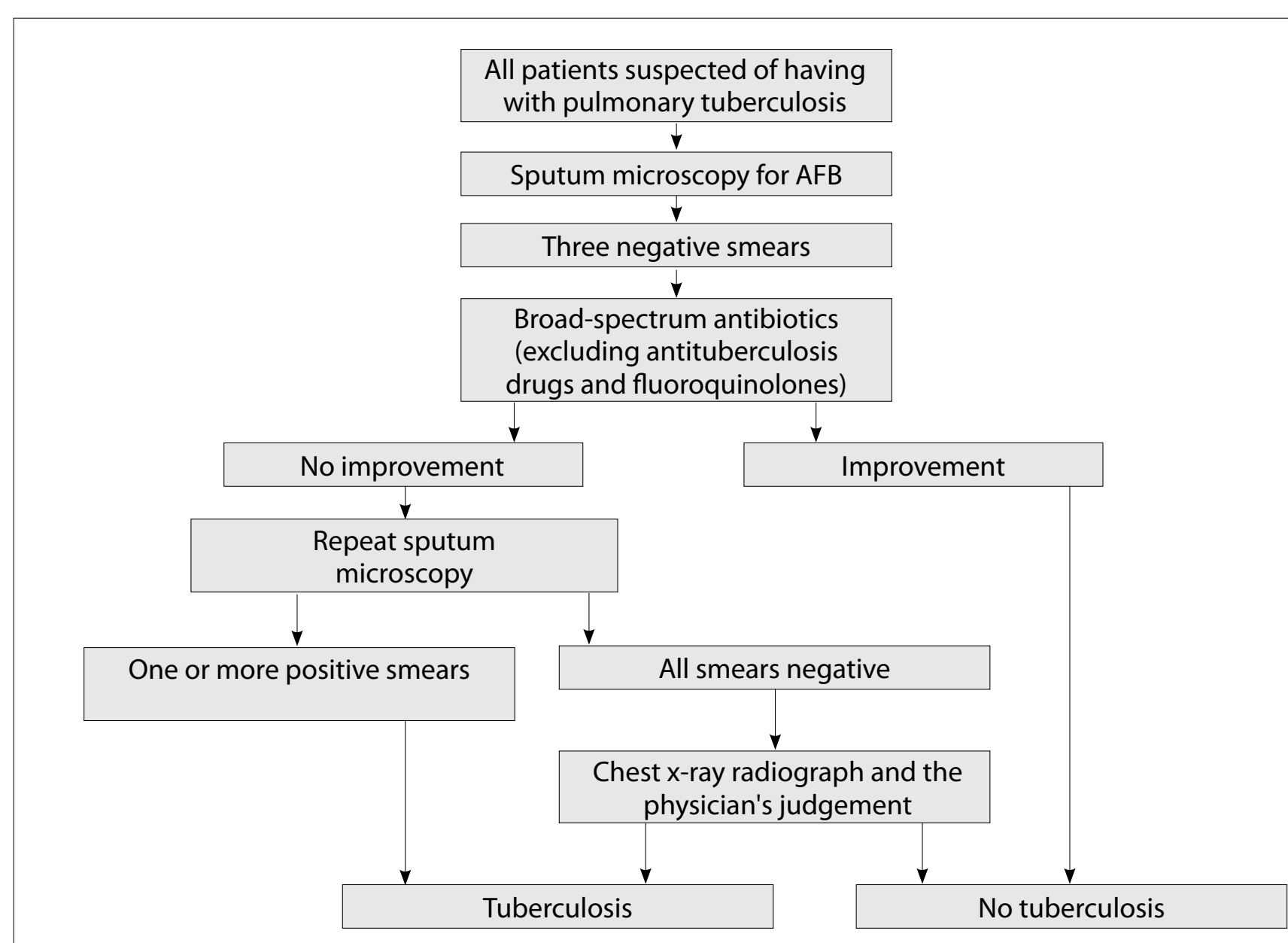

Figure 1. Algorithm of smear-negative tuberculosis diagnosis in HIV-negative patients (4) 
and cavitation is observed less frequently compared to smear-positive pulmonary tuberculosis patients (9). Thus, contagiousness of SNPT cases is lower. Although approximately $70 \%$ of smear-positive pulmonary tuberculosis cases die in 10 years when there is no treatment, this rate is $20 \%$ for SNPT cases (1). According to these results, the mortality rate of SNPT is lower than of smear-positive pulmonary tuberculosis; however, patients with SNPT have high mortality rates and must be diagnosed and treated immediately.

Smear-negative pulmonary tuberculosis can be confused with many diseases because of diagnostic problems and therefore, its differential diagnosis is very important (9). Especially, it is very important to make a differential diagnosis of pneumonia and other pulmonary pathologies. In the differential diagnosis of SNPT, bacterial pneumonia, interstitial pneumonias, pulmonary nocardiosis, pneumocystis jiroveci pneumonia, cryptococcal pneumonia, and histoplasmosis are included.

\section{Diagnosis}

\section{A. Microbiology}

Isolation of Mycobacterium tuberculosis is the "golden standard" in tuberculosis diagnosis. Sputum, gastric juice, bronchial lavage, pleural fluid, tissue biopsy samples, synovial fluid, cerebrospinal fluid (CSF), and urine taken for this purpose are investigated for bacillus. Microscopic investigation of sputum is still the most commonly used method for tuberculosis diagnosis. Typically, the Ziehl-Neelsen procedure (Carbolfuchsin method) is used in the microscopic investigation of sputum smears. The sensitivity of the microscopic investigation of sputum is $20-60 \%$ and $\geq 80 \%$ in some cases (10). Instead of direct microscope, sensitivity is increased by methods such as processing of the phlegm with centrifuge and chemicals such as $\mathrm{NaOH}$ before smear investigations (10). Although microscopic investigation is the fastest diagnostic method present, its sensitivity is relatively low. For a positive result, samples must contain more than 10.000 bacilli per milliliter. The diagnostic success of the microscopic investigation depends on the prevalence of the disease, presence of cavity, and the quality of the sample. Sputum smears give a negative result for approximately half of the pulmonary tuberculosis patients. Causes of negative results of the sputum smear include insufficiency or mistakes during taking, collection, processing, or investigation of sputum (11). Accurate results can be obtained by improvements and caution. For the patients who cannot take out sputum, sputum induction, bronchoscopy, or gastric juice collection must be considered. The sensitivity of standard microscopy can be increased by $10 \%$ with fluorescent microscopy (12). Another advantage of fluorescent microscopy is that samples can be evaluated faster. Light emitting diode (LED) fluorescent microscopy is a valid alternative to traditional methods (short arc mercury lamp) and its low cost creates an important advantage (13).

Culture is still the reference standard method for pulmonary tuberculosis diagnosis. In the presence of 10-100 bacilli in a milliliter of the sample and culture, a positive result can be obtained. The sensitivity of sputum culture is around $81.5 \%$ and the specificity is around $98.4 \%$ (14). Solid mediums such as Löwenstein-Jensen or liquid mediums such as Middlebrook $\mathrm{H} 12$ are used for culture. In recent years, methods such as BACTEC 460TB, BACTEC MB9600, and MGIT, which provide rapid results, are also used. WHO suggests the use of both solid and liquid mediums for the culture of samples (1). An advan- tage of the culture apart from sensitivity and specificity is that it enables the identification of species, the test for drug resistance, and gene typing for epidemiological studies. Microbiological culture results are obtained in approximately 2-6 weeks. Because the culture results are obtained after a longer time for patients with a pre-diagnosis of SNPT, clinicians remain undecided on whether to initiate tuberculosis treatment. Thirty-eight percent of pulmonary tuberculosis patients are smear- and culture-positive, $48 \%$ are smear-negative and culture-positive, and $14 \%$ are smear-negative and culture-negative (11). All advanced investigations, including high-resolution CT (HRCT) of the thorax and bronchoscopy, should be performed for patients with SNPT who are culture-negative.

Routine usage of bronchoscopy for the diagnosis of SNPT is controversial. In most of the guidelines, there are no direct suggestions regarding this. Bronchoscopy is suggested as a support procedure for the diagnosis of SNPT $(15,16)$. Especially, when it is used with thoracic HRCT, its sensitivity is increased in SNPT patients (17). Postbronchoscopic sputum sampling both helps the diagnosis and increases the culture positivity by $7 \%$ (18). However, in the same study, increased contamination was observed in one-third of the patients from whom phlegm was taken after bronchoscopy. On the samples taken with bronchoscopy, nucleic acid amplification tests (NAATs) and investigations with Xpert MTB/RIF can be performed $(19,20)$.

\section{B. Clinical Guess Score}

A large number of patients who are given antituberculosis treatment because of pulmonary tuberculosis cannot be diagnosed bacteriologically with smear and culture despite advanced diagnostic opportunities. Clinicians experience difficulty in deciding whether to initiate the treatment for patients with a pre-diagnosis of pulmonary tuberculosis because it takes a long time to obtain culture results if the smear is negative. Antituberculosis treatment treats the disease, prevents its progress, and decreases the contamination. On the other hand, because of the possibility of a misdiagnosis and potential side effects of the medication, clinicians hesitate to start treatment.

To ease the diagnosis of SNPT, the clinical score and/or guess systems and a variety of management algorithms were developed, but very few of them were validated. Most of the studies were conducted in populations with a high HIV prevalence. Moreover, local differences between countries are present. Samb et al. (21) revealed that for patients with a pre-diagnosis of SNPT who were hospitalized, the symptoms associated with tuberculosis were (1) cough ( $>21$ days), (2) chest pain (>15 days), (3) absence of sputum, and (4) absence of dyspnea. The presence of any two of these four symptoms was found to have $85 \%$ sensitivity and $67 \%$ specificity. In this study, the prevalence of HIV was high in patient and control groups (71\%) and only hospitalized patients were included. Kanaya et al. (22) developed a tuberculosis prediction score (TPS) by identifying clinical, demographic, and radiological characteristics of patients. TGS is used for patients whose cultures are going to result positive among SNPT patients. Tuberculin skin test (TST) positivity is independently together with increased culture-positivity and in contrast, radiological non-typical findings for tuberculosis and cough with sputum are indicators of decreased culture positivity (Table 1). In places where the tuberculosis prevalence is high, a conservative threshold value of TGS $\geq 0$ should be used for tuberculosis treatment. In places where tuberculosis prevalence is low, waiting is required, instead of starting the empirical treatment, until the culture results are obtained. This study that was conducted 
Table 1. Prediction system of tuberculosis in smear-negative lung tuberculosis (22)

\begin{tabular}{|c|c|c|c|c|}
\hline Predictors & $\begin{array}{c}\text { Multivariant OR } \\
(95 \% \mathrm{Cl})\end{array}$ & $\begin{array}{c}p \\
\text { value }\end{array}$ & Score & LR \\
\hline & & & -2 & 0.2 \\
\hline \multirow[t]{2}{*}{ Expectoration } & $0.3(0.1-0.6)$ & 0.002 & -1 & 0.5 \\
\hline & & & 0 & 1.8 \\
\hline TST positivity & $4.8(2.0-11.9)$ & 0.001 & +1 & 7.1 \\
\hline $\begin{array}{l}\text { Atypical infiltration } \\
\text { for TB }\end{array}$ & $0.3(0.1-0.7)$ & 0.006 & -1 & \\
\hline $\begin{array}{l}\text { HIV positivity and } \\
\text { mediastinal } \\
\text { lymphadenopathy }\end{array}$ & $7.2(1.4-36.0)$ & 0.02 & +2 & \\
\hline
\end{tabular}

HIV: Human immunodeficiency virus; LR: likelihood ratio; OR odds ratio; TB: tuberculosis; TST: tuberculin skin test

in a society with a low HIV and tuberculosis prevalence can also be suitable for Turkey. Various algorithms based on local epidemiological data and clinical score systems were developed for the diagnosis of SNPT (23). Most of them were validated only locally and not used commonly. However, in countries where SNPT is a serious public health issue, these algorithms can set a starting point for the development of diagnostic rules specific to that society and contribute to national tuberculosis programs (23).

\section{Auxiliary Tests}

Most SNPT patients are diagnosed on the basis of clinical and radiological findings. Laboratory tests for the diagnosis or for aiding diagnosis are also used. TST shows the infection, not the disease. TST can give positive result because of the sensitivity to atypical mycobacteria in societies using the BCG vaccine. Interferon gamma emission releasing assays (IGRAs) that have become common lately and do not get affected by BCG are also not suggested for the diagnosis of the active disease. IGRA is suggested in Turkey for patients who are TST negative (with booster), who are highly suspected of a tuberculosis infection, who have suppressed immunity, or who are candidates for suppressive treatment (3). Nucleic acid amplification test has low sensitivity (40.4-44.2\%) especially in smear-negative samples (24). Gene Xpert is a new molecular test recently used in tuberculosis treatment frequently. This test is suggested by WHO for HIV-positive patients or for patients suspected of tuberculosis resistant to drugs (1). In SNPT patients, the sensitivity of Gene Xpert for one sample is $50-70 \%$ and it increases with the sample number (25). Positivity of the Gene Xpert test has been found to be related to the basil load in the sample (26). Sensitivity of Anda-TB IgG, which is a serological diagnostic test developed in recent years, in smear-positive patients is $63-85 \%$ and its specificity is $73-100 \%$ and no sufficient information about the use of this test in smear-negative patients is available (27). WHO suggests not using serological tests in pulmonary and extrapulmonary tuberculosis diagnosis because of the inconsistency and impreciseness of their sensitivity and specificity values (28).

\section{Radiology}

Radiology is an extremely valuable diagnostic tool for SNPT. The radiological findings of SNPT patients are different from those of smear-positive patients. Radiological changes in SNPT patients are less significant and cavitation is less frequently seen (23). The specificity of chest X-ray in pulmonary tuberculosis diagnosis is high (78\%) and its sensitivity is low (51\%) (29). The low sensitivity of the chest X-ray limits its usage. Radiological findings differ between primary tuberculosis and secondary (reactivation, postprimary) pulmonary tuberculosis. In primary tuberculosis, parenchymal disease, lymphadenopathy (43-96\%), miliary disease (1-7\%), and pleural effusion (25\%) are seen most commonly (30). In postprimary tuberculosis, parenchymal disease (consolidation, cavity), airway involvement (10-40\%), and pleural involvement (18\%) are observed more commonly (30). Pulmonary tuberculosis typically involves the apical and posterior segments of the upper lobes in $85 \%$ of patients. In a lower rate, tuberculosis is detected in the anterior segment of the upper lobe and anterior segment of the lower lobe. Infiltration and cavity screenings are seen typically on chest X-rays. Cavities seen in pulmonary tuberculosis are generally thin walled. Acinar infiltration and pneumonia can arise in the lower lobes in association with endobronchial spread. HRCT enables the lesions that cannot be seen on chest X-ray to be monitored (31). Based on the HRCT findings, guessing systems that predict sputum smear positivity have been previously tried to be developed (32). In SNPT patients, many, lesions have been identified on HRCT. By evaluating radiological findings and localizations in these patients, it has been suggested that HRCT can be used for guessing patients with high risk of SNPT (33). The most common radiological findings detected on HRCT in SNPT patients are centrilobular nodules (73\%), nodules (71\%), and consolidation (54\%) (34).

\section{Treatment}

In Tuberculosis Diagnosis and Treatment Guideline published in 2011, the quadruple therapy of $\mathrm{H}$ (isoniazid), R (rifampicin), Z (pyrazinamide), and $\mathrm{E}$ (ethambutol) is recommended for 2 months at the beginning of SNPT treatment. Subsequently, during the maintenance period, the dual therapy of HR is recommended for 4 months and standard antituberculosis treatment is recommended for 6 months (3). In the tuberculosis treatment guideline published by WHO in 2010, the suggested treatment regime, including triple therapy of HRZ for 2 months and dual therapy of HR for 4 months, was switched to quadruple therapy of HRZE for 2 months, dual therapy of HR for 4 months, and standard treatment for 6 months (35).

\section{CONCLUSION}

Smear-negative pulmonary tuberculosis is still one of the most problematic issues of tuberculosis. In preliminary diagnosed SNPT patients, microbiological samples (sputum, induced sputum, gastric juice, bronchoscopy, and bronchoalveolar lavage, when necessary) should be taken, radiological investigations (chest X-ray, HRCT included, where necessary) should be performed, elimination of the diseases included in the differential diagnosis should be made, and antituberculosis treatment should be initiated following the diagnosis of SNPT established by experienced centers after completion of all these procedures. In recent years, important developments in the diagnosis of SNPT have been reached with advanced radiological examinations such as HRCT and new microbiological diagnostic methods. In our country, new studies that can create clinical guessing score systems and algorithms, including clinical, laboratory, and radiology, and the development of diagnostic tests with high sensitivity are warranted. 
Peer-review: Externally peer-reviewed.

Author contributions: Concept - T.Ç., H.K.; Design - T.Ç., H.K.; Supervision T.Ç., H.K.; Resource - T.Ç., H.K.; Materials - T.Ç., H.K.; Data Collection and/or Processing - T.Ç., H.K.; Analysis and/or Interpretation - T.Ç., H.K.; Literature Search - T.Ç., H.K.; Writing - T.Ç., H.K.; Critical Reviews - T.Ç., H.K.; Other - T.Ç., H.K.

Conflict of Interest: No conflict of interest was declared by the authors.

Financial Disclosure: The authors declared that this study has received no financial support.

\section{REFERENCES}

1. Global Tuberculosis Report 2013, World Health Organization, Geneva Switzerland.

2. Türkiye'de Verem Savaşı 2012 Raporu, T.C. Sağlık Bakanlığı Türkiye Halk Sağlığı Kurumu Tüberküloz Daire Başkanlığı, 884, Ankara 2013.

3. Tüberküloz Tanı ve Tedavi Rehberi, T.C. Sağlık Bakanlığı, 862, Ankara 2011.

4. Improving the diagnosis and treatment of smear-negative pulmonary and extrapulmonary tuberculosis among adults and adolescents, World Health Organization, 2007.

5. Tostmann A, Kik SV, Kalisvaart NA, Sebek MM, Verver S, Boeree MJ, et al. Tuberculosis transmission by patients with smear-negative pulmonary tuberculosis in a large cohort in the Netherlands. Clin Infect Dis 2008; 47: 1135-42. [CrossRef]

6. Behr MA, Warren SA, Salamon H, Hopewell PC, Ponce de Leon A, Daley $\mathrm{CL}$, et al. Transmission of Mycobacterium tuberculosis from patients smear-negative for acid-fast bacilli. Lancet 1999; 353: 444-9. [CrossRef]

7. Thapa B. Smear negative pulmonary tuberculosis and infectivity. Int J Infect Microbiol 2013; 2: 68-9. [CrossRef]

8. Murray CJ, Styblo K, Rouilon A. Tuberculosis in developing countries: burden, intervention and cost. Bull Int Union Tuberc Lung Dis 1990; 65: 6-24.

9. Colebunders R, Bastian I. A review of the diagnosis and treatment of smear-negative pulmonary tuberculosis. Int J Tuberc Lung Dis 2000; 4: 97-107.

10. Steingart KR, Ng V, Henry M, Hopewell PC, Ramsay A, Cunningham J, et al. Sputum processing methods to improve the sensitivity of smear microscopy for tuberculosis: a systematic review. Lancet Infect Dis 2006; 6: 664-74. [CrossRef]

11. Long R. Smear-negative pulmonary tuberculosis in industrialized countries. Chest 2001; 120: 330-4. [CrossRef]

12. Steingart KR, Henry M, Ng V, Hopewell PC, Ramsay A, Cunningham J, et al. Fluorescence versus conventional sputum smear microscopy for tuberculosis: a sytematic review. Lancet Infect Dis 2006; 6: 570-81. [CrossRef]

13. Marais BJ, Brittle W, Painczyk K, Hesseling AC, Beyers N, Wasserman E, et al. Use of light-emitting diode fluorescence microscopy to detect acidfast bacilli in sputum. Clin Infect Dis 2008; 47: 203-7. [CrossRef]

14. Levy H, Feldman C, Sacho H, van der Meulen H, Kallenbach J, Koornhof $\mathrm{H}$. A reevaluation of sputum microscopy and culture in the diagnosis of pulmonary tuberculosis. Chest 1989; 95: 1193-7. [CrossRef]

15. Quaiser S, Aqarwal A, Khan R, Haque SF. Fiberoptic bronchoscopy, as a valuable diagnostic option in sputum negative pulmonary tuberculosis: A prospective study. Int J Appl Basic Med Res 2012; 2: 123-7. [CrossRef]

16. Chandra TJ, Dash S, Srinivas G, Rao PV. A study on rapid confirmation of pulmonary tuberculosis in smear-negative acid fast bacilli cases by using fiberoptic bronchoscopy, done through oropharyngeal spacer. J Family Community Med 2012; 19: 43-6. [CrossRef]
17. Shin JA, Chang YS, Kim TH, Kim HJ, Ahn CM, Byun MK. Fiberoptic bronchoscopy for rapid diagnosis of smear-negative pulmonary tuberculosis. BMC Infect Dis 2012; 12: 141. [CrossRef]

18. George PM, Mehta M, Dhariwal J, Singanayagam A, Raphael CE, Salmasi M, et al. Post-bronchoscopy sputum: improving the diagnostic yield in smear-negative pulmonary TB. Respir Med 2011; 105: 1726-31. [CrossRef]

19. Lee HY, Seong MW, Park SS, Hwang SS, Lee J, Park YS, et al. Diagnostic accuracy of Xpert MTB/RIF on bronchoscopy specimens in patients with suspected pulmonary tuberculosis. Int JTuberc Lung Dis 2013; 17: 917-21. [CrossRef]

20. Boonsarnqsuk V, Suwannaphong S, Laohavich C. Combination of adenosine deaminase activity and polymerase chain reaction in bronchoalveolar lavage fluid in the diagnosis of smear-negative active pulmonary tuberculosis. Int J Infect Dis 2012; 16: e663-8. [CrossRef]

21. Samb B, Henzel D, Daley CL, Muqusi F, Niyangabo T, Mika-Cabanne N, et al. Methods for diagnosing tuberculosis among in-patients in estern Africa whose sputum smears are negative. Int J Tuberc Lung Dis 1997; 1:25-30.

22. Kanaya AM, Glidden DV, Chambars HF. Identifying pulmonary tuberculosis in patients with negative sputum smear results. Chest 2001; 120 : 349-55. [CrossRef]

23. Siddiqi K, Lambert ML, Walley J. Clinical diagnosis of smear-negative pulmonary tuberculosis in low-income countries: the current evidence. Lancet Infect Dis 2003; 3: 288-96. [CrossRef]

24. Ito K, Yoshiyama T, Nakazono, Oqata H, Wada M, Mizutani S. Limits of nucleic acid amplification tests for smear negative pulmonary tuberculosis. Kekkaku 2000; 75: 691-7.

25. Boehme CC, Nabeta P, Hillemann D, Nicol MP, Shenai S, Krapp F, et al. Rapid molecular detection of tuberculosis and rifampin resistance. $\mathrm{N}$ Engl J Med 2012; 363: 1005-15. [CrossRef]

26. Theron G, Peter J, van Zyl-Smit R, Mishra H, Streicher E, Murray S, et al. Evaluation of the Xpert MTB/RIF assay fort he diagnosis of pulmonary tuberculosis in a high HIV prevalence setting. Am J Respir Crit Care Med 2011; 184: 132-40. [CrossRef]

27. Steingart KR, Henry M, Laal S, Hopewell PC, Ramsay A, Menzies D, et al. Commercial serological antibody detection tests fort he diagnosis of pulmonary tuberculosis: a sytematic review. PLoS Med 2007; 4: e202. [CrossRef]

28. Commercial serodiagnostic tests for diagnosis of tuberculosis, expert group meeting report 2010, WHO 2011, Geneva, Switzerland.

29. Kumar N, Bhargava SK, Agrawal CS, George K, Karki P, Baral D. Chest radiographs and thier reliability in the diganosis of tuberculosis. JNMA J Nepal Med Assoc 2005; 44: 138-42.

30. Burrill J, Williams CJ, Bain G, Conder G, Hine AL, Misra RR. Tuberculosis: a radiologic review. Raidographics 2007; 27: 1255-73. [CrossRef]

31. Wang YH, Lin AS, Lai YF, Chao TY, Liu JW, Ko SF. The high value of high-resolution computed tomography in predicting the activity of pulmonary tuberculosis. Int J Tuberc Lung Dis 2003; 7: 563-8.

32. Yeh JJ, Yu JK, Teng WB, Chou CH, Hsieh SP, Lee TL, et al. High-resolution $\mathrm{CT}$ for identify patients with smear-positive, active pulmonary tuberculosis. Eur J Radiol 2012; 81: 195-201. [CrossRef]

33. Nakanishi M, Demura Y, Ameshima S, Kosaka N, Chiba Y, NishikawaS, et al. Utility of high-resolution computed tomography for predicting risk of sputum smear-negative pulmonary tuberculosis. Eur J Radiol 2010; 73: 545-50. [CrossRef]

34. Tozkoparan E, Deniz O, Ciftci F, Bozkanat E, Bıcak M, Mutlu H, et al. The roles of $\mathrm{HRCT}$ and clinical parameters in assessing activity os suspected smear negative pulmonary tuberculosis. Arch Med Res 2005; 36: 166-70. [CrossRef]

35. Treatment of Tuberculosis Guidelines, fouth edition, World Health Organization, 2010, Geneva, Switzerland. 